\title{
Characterization of sphere-forming cells with stem-like properties from the gastric cancer cell lines MKN45 and SGC7901
}

\author{
XUMING WANG ${ }^{1,2^{*}}$, FENG ZOU ${ }^{1,2^{*}}$, HAO DENG $^{1,2}$, ZHENGQI FU $^{1,2}$, \\ YAN LI ${ }^{1,2}$, LIXIA WU ${ }^{3}$, ZHAOYI WANG ${ }^{1,2}$ and LIJIANG LIU ${ }^{1,2}$ \\ ${ }^{1}$ Department of Pathology and Pathophysiology, School of Medicine; ${ }^{2}$ Jiangda Pathology Institute, \\ Jianghan University, Wuhan, Hubei 430056; ${ }^{3}$ Department of Pathology and Pathophysiology, \\ School of Basic Medical Science of Wuhan University, \\ Wuhan, Hubei 430072, P.R. China
}

Received November 30, 2013; Accepted April 28, 2014

DOI: $10.3892 / \mathrm{mmr} .2014 .2601$

\begin{abstract}
Traditionally, it was presumed that gastric cancer was derived from tumor cells with stem-like properties. In the present study, stem-like cells from the gastric cancer cell lines MKN45 and SGC7901 were enriched by growing them as spheres in a defined serum-free medium. Following enrichment for stem-like cells, cluster of differentiation (CD)24 and CD44 were applied as candidate stem cell markers to examine the expression profile. It was revealed that the sphere-derived cells contained a higher proportion of cells expressing the stem cell surface markers CD24 and CD44 when compared with the parental cells. It was also identified that the expression of cytokeratin 18 in sphere-derived cells was decreased and the expression of vimentin and aldehyde dehydrogenase 1 (ALDH1) was increased compared with the parental cells. This finding supports the existence of a population of tumor sphere-forming cells with stem cell properties in the MKN45 and SGC7901 cell lines. Furthermore, the stem cell population was enriched in cells expressing CD24, CD44, vimentin and ALDH1 cell surface markers. These results support the existence of gastric cancer stem cells and provide an alternative approach to the diagnosis and treatment of gastric cancer.
\end{abstract}

\section{Introduction}

Gastric cancer is one of the most common types of cancer worldwide with $\sim 989,600$ new cases and 738,000 mortalities annually, accounting for $\sim 8 \%$ of all new cancers (1-4).

Correspondence to: Professor Lijiang Liu, Department of Pathology and Pathophysiology, School of Medicine, Jianghan University, 8 Sanjiaohu Road, Wuhan, Hubei 430056, P.R. China E-mail: liulijiang@163.com

${ }^{*}$ Contributed equally

Key words: gastric cancer, sphere-forming cell, stem-like property
Common therapeutic methods have not been able to decrease the high mortality rate of gastric cancer. Gastric cancer is heterogeneous in nature and visible via scan/X-ray although the association between its histological phenotype and its biological behavior remains to be elucidated. Recently, a cancer stem cell hypothesis was proposed to explain the heterogeneity of cancer cells. The cancer stem cell hypothesis, in contrast to conventional stochastic theory, hypothesizes that only cancer stem cells (CSCs) are able to initiate tumor formation, self-renewal and differentiation. CSCs have been verified to drive the growth and metastasis of cancer cells (2). In order to investigate the mechanisms underlying the behavior of gastric cancer, the establishment of an in vitro model of CSCs is required.

Previous studies have demonstrated that CSCs existed in the gastric cancer cell lines MKN-45, MKN-74 and NCI-N87, which can be identified by the cluster of differentiation (CD)44 marker (5). The CD $44^{+}$gastric cancer cells possessed typical stem cell properties, including self-renewal and the ability to form differentiated progeny giving rise to CD44 cells (5). Furthermore, CD24 and epithelial cell adhesion molecule (EpCAM) were used as the gastric cancer stem cell markers $(6,7)$.

In the present study, CSCs were isolated from human gastric cancer cell lines (MKN-45 and SGC7901) using tumorsphere culturing in serum-free medium and the expression of CSC markers CD44 and CD24 were identified. Furthermore, the expression of cytokeratin 18 (CK18), vimentin and aldehyde dehydrogenase 1 (ALDH1) was also examined in order to investigate the characteristics of CSCs.

\section{Materials and methods}

Reagents. Dulbecco's modified Eagle's medium (DMEM)/high glucose, DMEM/F12 and B27 were obtained from Gibco-BRL (Grand Island, NY, USA). Epidermal growth factor (EGF), basic fibroblast growth factor (bFGF) and glucocorticoid were obtained from PeproTech, Inc. (Rocky Hill, NJ, USA). The anti-CD24 antibody (bs-0528R) and the anti-CD44 antibody (bs-0521R) were obtained from Beijing Biosynthesis Biotechnology Co., Ltd. (Beijing, China). The anti-CK18 
antibody (BM0032) and the anti-vimentin antibody (BM0135) were obtained from Wuhan Boster Biological Technology, Ltd. (Wuhan, China). The anti-ALDH1 antibody (611194) was obtained from BD Transduction Laboratories (San Diego, CA, USA). The mouse anti- $\beta$-actin monoclonal antibody (sc-47778) was obtained from Santa Cruz Biotechnology, Inc., Santa Cruz, CA, USA). The fluorescein-conjugated immunopure goat anti-rabbit IgG (ZF-0312) and the fluorescein-conjugated immunopure goat anti-mouse IgG (ZF-0311) were obtained from Pierce Biotechnology, Inc. (Rockford, IL, USA). The anti- $\beta$-actin antibody (sc-47778) was obtained from Santa Cruz Biotechnology, Inc. The appropriate secondary antibodies [goat anti-mouse IgG-HRP (sc-2005) and goat antirabbit IgG-HRP (sc-2004)] were obtained from Santa Cruz Biotechnology, Inc. Radioimmunoprecipitation assay buffer and an Enhanced BCA Protein Assay kit were purchased from Beyotime Institute of Biotechnology (Shanghai, China). Polyvinylidene fluoride (PVDF) membranes were obtained from Millipore (Billerica, MA, USA).

Cell lines. The human gastric cancer cell line MKN45 and SGC7901 were obtained from the Chinese Academy of Medical Sciences Cell Center of Basic Medicine (Beijing, China).

Cell line and sphere culture. MKN45 and SGC7901 cell lines were maintained in DMEM containing 10\% fetal bovine serum (Invitrogen Life Technologies, Carlsbad, CA, USA) at $37^{\circ} \mathrm{C}$ in a $5 \% \mathrm{CO}_{2}$ atmosphere. To obtain spheres in culture, the cells were plated at a density of $10^{5}$ cells $/ \mathrm{ml}$ in serum-free medium (DMEM/F12; Invitrogen Life Technologies) supplemented with $20 \mathrm{ng} / \mathrm{ml} \mathrm{bFGF}, 20 \mathrm{ng} / \mathrm{ml}$ EGF, $0.5 \mu \mathrm{g} / \mathrm{ml}$ glucocorticoid and 2\% B27. Culture medium was replaced or supplemented with additional growth factors every 4 days. To propagate spheres in vitro, sphere cells were collected by gentle centrifugation at $150 \mathrm{x}$ g for $3 \mathrm{~min}$, dissociated into single-cell suspensions and cultured to allow the regeneration of spheres. Second-generation spheres were used for all subsequent experiments. The total number of tumor spheres was counted following 9 days of culture. Sphere formation efficiency was calculated within 10 high power fields. The sphere from the MKN45 cell line was fixed in 10\% neutral formalin, then coated onto glass slides and stained with hematoxylin and eosin. Subsequently, the cells were observed using a fluorescence microscope (magnification, $\mathrm{x} 400$; Olympus, Tokyo, Japan).

Western blot analysis. For western blot analysis, the cells were washed with cold phosphate-buffered saline (PBS) and lysed with the lysis buffer [50 mM Tris- $\mathrm{HCl} \mathrm{pH} 8.0,150 \mathrm{mM}$ $\mathrm{NaCl}, 0.25 \mathrm{mM}$ ethylenediaminetetraacetic acid $\mathrm{pH} 8.0$, $0.1 \%$ sodium dodecyl sulfate (SDS), $1 \%$ Triton X-100, $50 \mathrm{mM}$ $\mathrm{NaF}]$ supplemented with protease and phosphatase inhibitors purchased from Sigma (St. Louis, MO, USA). The protein concentrations were determined using an Enhanced BCA Protein Assay kit (Beyotime Institute of Biotechnology). Cell lysates were mixed with loading buffer (Beyotime Institute of Biotechnology), separated by $12 \%$ SDS-polyacrylamide gel and transferred onto a PVDF membrane $(0.22 \mu \mathrm{m}$; Millipore $)$. The membranes were probed with various primary antibodies (the mouse anti-CK18 monoclonal antibody, the mouse anti-vimentin monoclonal antibody, the mouse anti-ALDH1 monoclonal antibody and the mouse anti- $\beta$-actin monoclonal antibody) and appropriate secondary antibodies (the goat anti-mouse IgG-HRP and the goat anti-rabbit IgG-HRP), and visualized with enhanced chemiluminescence (ECL; Beyotime Institute of Biotechnology) detection reagents (DNR Bio-Imaging Systems, Jerusalem, Israel). The densities of protein bands were assessed using TotalLab analysis software (Nonlinear Dynamics, Durham, NC, USA).

Immunofluorescence staining and flow cytometry (FCM). For immunofluorescence staining, cells were collected in a $1.5 \mathrm{ml}$ centrifuge tube, fixed with $4 \%$ paraformaldehyde for $30 \mathrm{~min}$ at room temperature, then coated onto glass slides and permeabilized with $0.1 \%$ Triton X-100/PBS for $15 \mathrm{~min}$ at room temperature and then incubated with the Hoechst 33258 (Beyotime Institute of Biotechnology) for $10 \mathrm{~min}$. Subsequently, the cells were observed using a fluorescence microscope (magnification, $x 400$; Olympus). For FCM analyses, tumor spheres were dissociated as single cells, washed and incubated with the appropriate concentration of control or specific antibody for $45 \mathrm{~min}$. Then, the cells were washed and incubated with CD44 and CD24 antibodies for $1 \mathrm{~h}$, and then with fluorescein isothiocyanate-conjugated secondary antibodies for $30 \mathrm{~min}$. All samples were measured using a flow cytometer (BD Biosciences, Franklin Lakes, NJ, USA).

Statistical analysis. The statistical analysis was performed using SPSS 12.0 software (SPSS, Inc., Chicago, IL, USA). The results are shown as the mean \pm standard deviation in three replicate samples and compared using Student's t-test and analysis of variance. $\mathrm{P}<0.05$ was considered to indicate a statistically significant difference. All experiments were performed at least three times to ensure reproducibility of the results.

\section{Results}

Sphere formation and counting. To determine if a population of CSCs exists in gastric cancer cells lines, the gastric cancer cells lines MKN45 and SGC7901 were grown in serum-free sphere-promoting conditions. Following 9 days of culture, golf ball-like spheres were observed ranging between 40 and 100 cells per sphere. Cells from the MKN45 and SGC7901 cell lines were able to form spheres, however, they produced different quantities, suggesting that each cell line contains a different number of stem-like cells. MKN45 cells exhibited a higher sphere-forming efficiency than the SGC7901 cells (Fig. 1).

Expression profile of cell surface markers. In order to further characterize the expression profile of cell surface markers on sphere-derived cells, FCM analysis and western blot analysis were performed on several candidate stem cells. The FCM analysis demonstrated that CD44 and CD24 was only partially expressed in the parental MKN45 and SGC7901 cells, however, this expression was increased in the MKN45 sphere cells and SGC7901 sphere cells (Figs. 2 and 3). Western blot analysis 
A
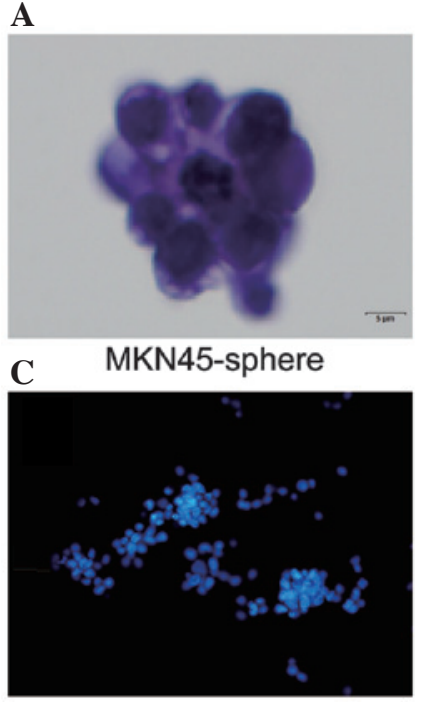

MKN45-sphere

E
B

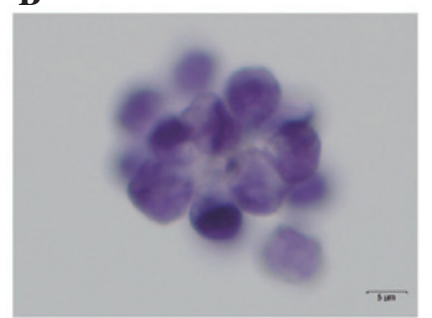

D SGC7901-sphere

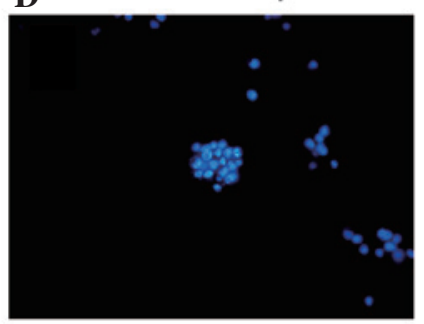

SGC7901-sphere

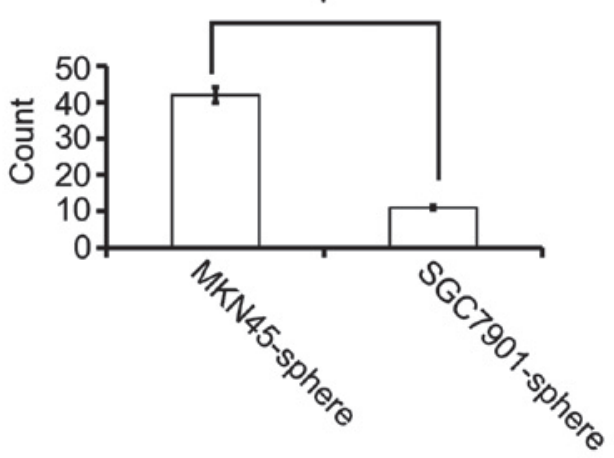

Figure 1. Formation of tumorsphere cells. (A) MKN45 tumorspheres stained with H\&E. (B) SGC7901 tumorspheres stained with H\&E. (C) MKN45 tumorsphere formation after 12 days. (D) SGC7901 tumorsphere formation after 12 days. Magnification, $x 400$. (E) Number of tumorspheres in 10 high power fields. The experiment was repeated three times. Data are presented as the mean \pm standard deviation of three independent experiments. ${ }^{*} \mathrm{P}<0.05$, compaared with the MKN45-spheres. H\&E, hematoxylin and eosin.

demonstrated that the expression of CK18 was reduced in the MKN45 sphere cells and SGC7901 sphere cells compared with in the parental MKN45 and SGC7901 cells. Western blot analysis also demonstrated that the expression of ALDH1 was increased in the MKN45 sphere cells and SGC7901 sphere cells compared with in the parental MKN45 and SGC7901 cells. In addition, no vimentin expression was observed in the parental MKN45 and SGC7901 cells, however, vimentin was partially expressed in the MKN45 sphere cells and SGC7901 sphere cells (Fig. 4).

\section{Discussion}

Gastric cancer is one of the most severe diseases worldwide. Despite advances in various diagnostic tools and therapies, the five-year relative survival rate remains extremely low (8-11). One century ago, Julius Cohnheim proposed that tumors are derived not from normal adult tissues, but from 'embryonal cell rests', which he described as residual embryonic cells 'left behind' in the adult organism (12). It was hypothesized that CSCs are important in tumor initiation, invasion, metastasis
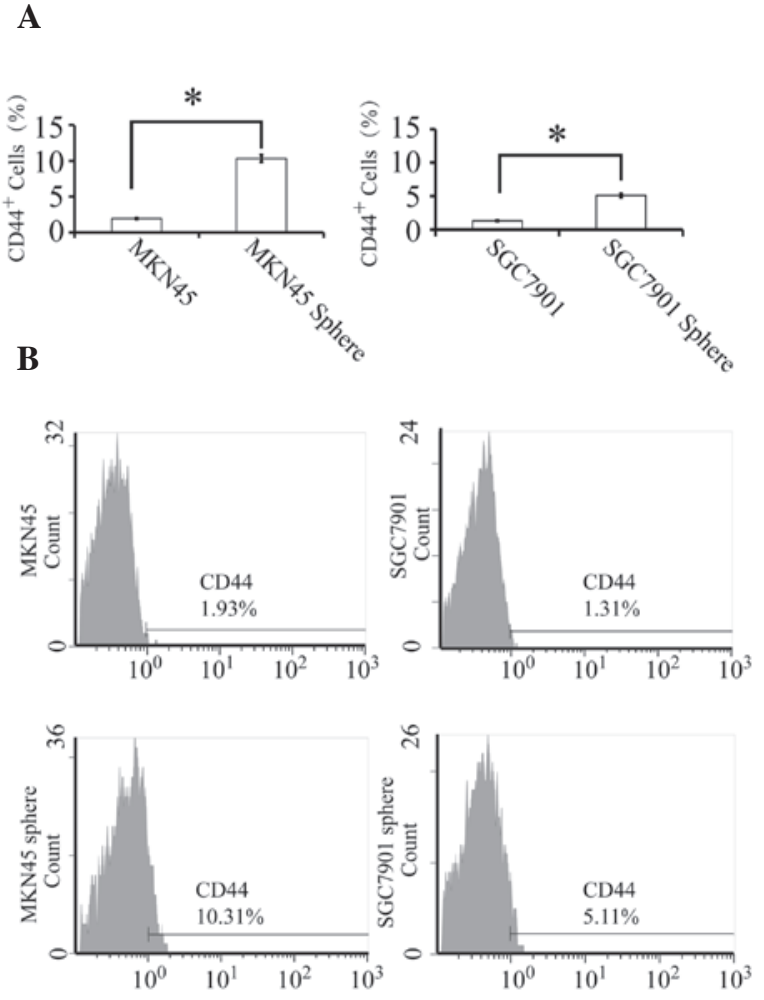

Figure 2. Expression of CD44 in parental cells and tumorsphere cells. (A) Flow cytometric analyses showing the increased percentages of CD44 ${ }^{+}$ cells in MKN45 spheres and in SGC7901 spheres when compared with the parental cells. ${ }^{*} \mathrm{P}<0.05$, compared with the parental cells. Data are presented as the mean \pm standard deviation of three independent experiments. (B) Flow cytometric analysis of CD44. CD, cluster of differentiation.
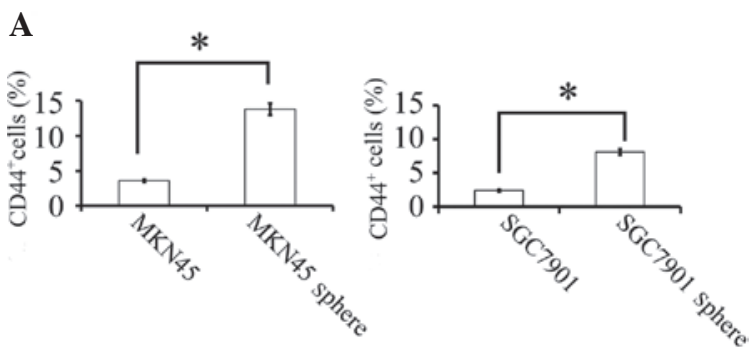

B
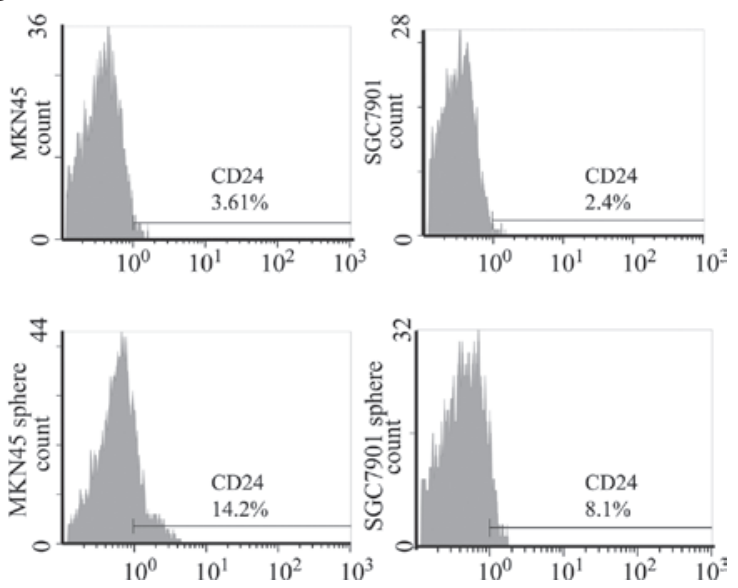

Figure 3. Expression of CD24 in parental cells and tumorsphere cells. (A) Flow cytometric analyses showing the increased percentages of CD24 cells in MKN45 spheres and in SGC7901 spheres when compared with the parental cells. $\mathrm{P}<0.05$, compared with the parental cells. Data are presented as the mean \pm standard deviation of three independent experiments.(B) Flow cytometric analysis of CD24. CD, cluster of differentiation. 


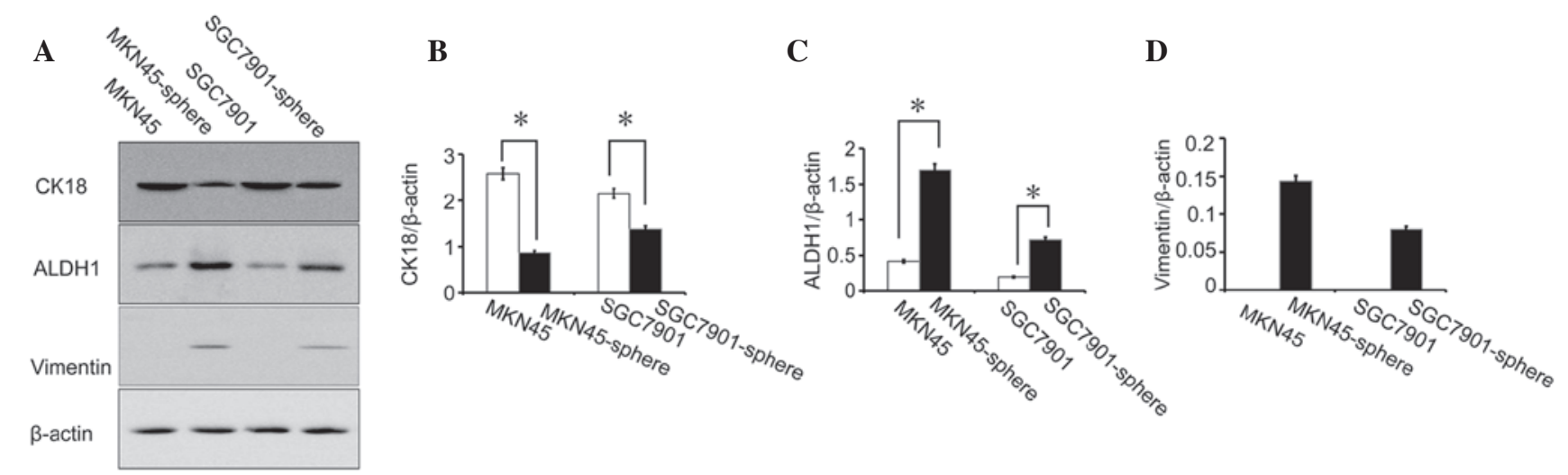

Figure 4. Expression of cancer stem cell markers in parental cells and tumorsphere cells. (A) Western blot analysis result showing CK18, ALDH1 and vimentin expression in parental cells and tumorsphere cells. (B) CK18 expression in MKN45 parental and tumorsphere cells and in SGC7901 parental and tumorsphere cells. (C) ALDH1 expression in MKN45 parental and tumorsphere cells and in SGC7901 parental and tumorsphere cells. (D) Vimentin expression in MKN45 parental and tumorsphere cells and in SGC7901 parental and tumorsphere cells. " $\mathrm{P}<0.05$ compared with the parental cells. Data are presented as the mean \pm standard deviation of three independent experiments. CK18, cytokeratin 18; ALDH1, aldehyde dehydrogenase 1.

and resistance to anticancer therapies (13). A previous study demonstrated that stem cell-like cells can be isolated in vitro from gastric cancer tissues, which suggested that CSCs existed in gastric cancer (14). The method of inducing parental cells into a spheroid body has been increasingly used for enriching stem cells and is dependent on their property of anchorage-independent growth. There were numerous types of applications in spheroid body culture used to isolate and enrich potential CSC subpopulations from primary tumors (15-17). It was reported that spheroid bodies were derived by placing the parental MKN45 cells into serum-free RPMI-1640 culture medium containing N-2, B27, antibiotic mixture, FGF-2 and EGF (18). In the present study, it was revealed that cells from the MKN45 and SGC7901 cell lines were able to form spheres in serum-free medium but produced different quantities and MKN45 cells exhibited a higher sphere-forming efficiency than SGC7901 cells.

Previous studies demonstrated that ALDH1, CD24, CD44, CD54, CD90, CD133, CD166 and EpCAM were stem cell markers in gastric cancer $(6,19,20)$. CD44 was regarded as the main gastric CSC marker, which was reported to be expressed in several studies of gastric CSCs $(5,21)$. Three gastric cancer cell lines, including MKN-74, MKN-45 and NCI-N87 had a substantial subpopulation of $\mathrm{CD} 44^{+}$cell. When these cells were injected into the stomach and skin of mice with severe combined immunodeficient (SCID) in vivo, spheroid colony formation was observed in serum-free media in vitro and the cells possessed tumorigenic ability. The $\mathrm{CD} 44^{+}$gastric cancer cells exhibited the characteristic stem cell properties of self-renewal and the ability to form differentiated progeny and generate CD44- cells (5). Following CD44 knockdown using short hairpin RNA, spheroid colony formation was significantly reduced and tumor production in SCID mice decreased. In addition, the CD44- populations had significantly reduced tumorigenic ability in vitro and in vivo (5). The present study demonstrated that the expression of CD44 was higher in the MKN45 sphere cells and SGC7901 sphere cells than in the parental MKN45 and SGC7901 cells, which indicated that CD44 was an important stem cell marker.

CD24 is expressed in putative stem cells within numerous tissues and is often overexpressed in colonic and gastric adenocarcinomas (22). In certain studies, CD24 has been found to be a gastric stem cell marker (21,23-26). The number of floating tumorsphere cells expressing CSC markers, including CD44, CD24 and CD133, was significantly higher in tumorsphere cells compared with in adherent cells (7). However, the present study demonstrated that CD24 was more highly expressed in the MKN45 sphere cells and SGC7901 sphere cells than in the parental MKN45 and SGC7901 cells, indicating that CD24 was an important stem cell marker.

Mesenchymal stem cells (MSCs) have been isolated from various human tissues. MSCs have various characteristics which all contribute to their therapeutic potential, including their low immunogenicity, the ability to secrete cytokines and their plasticity. It has recently been reported that MSCs are also involved in tumorigenesis and its prognosis (14). Certain stem cell-associated genes, including Oct-4, Nanog, Bmi1, Nucleostemin, ABCG2, CD44, CD73, CD90 (Thy-1) and other genes, including $\alpha$-smooth muscle actin ( $\alpha$-SMA) and vascular endothelial growth factor were expressed by MSCs and SGC7901 cells (14). However, the expression of CD90 (Thy-1), $\alpha$-SMA and ING4 were significantly greater in MSCs than in SGC7901 cells (14). By contrast, CK18 was detected in SGC-7901 cells but not in MSCs. MSCs derived from human gastric cancer and human bone marrow were moderately positive for mutant p53 and CK18. SGC7901 cells were strongly positive for them $(14,27)$. The present study demonstrated that the expression of CK18 was decreased in the MKN45 sphere cells and SGC7901 sphere cells compared with that in the parental MKN45 and SGC7901 cells. Furthermore, the immunoblot analysis suggested that there was no vimentin expression in the parental MKN45 and SGC7901 cells, however, vimentin was partially expressed in the MKN45 sphere cells and SGC7901 sphere cells. The former demonstrated that the MKN45 sphere cells and SGC7901 sphere cells possessed the characteristics of MSCs.

ALDH1 has been demonstrated to serve as a marker for cancer-initiating cells. A previous study found that 5-8\% of human diffuse type gastric carcinoma cells consisted of ALDH1+ cells and that OCUM-2MLN and HSC-39 cells were more prone to developing cancer than that of ALDH1+ (28). 
Several other studies also indicated that cells, such as CD133+, CD26+, ALDH1+ and CD44+ cells were found in the stem cell zone close to the normal mucosa together with Lgr5+ cells and they were these markers were also detected on the surface of gastric cancer cells (29). The present study found that the expression of ALDH1 was higher in the MKN45 sphere cells and SGC7901 sphere cells than in the parental MKN45 and SGC7901 cells, indicating that ALDH1 was an important stem cell marker.

In conclusion, the present study succeeded in culturing gastric CSCs from the MKN45 and SGC7901 cells. Furthermore, the sphere cells possessed the typical characteristics of MSCs. The results may aid in the development of novel culture methods to produce gastric stem cells in vitro, which can be used to test potential therapeutic agents for gastric cancer.

\section{Acknowledgements}

This study was supported by the National Natural Science Foundation of China (grant no. 81272754 and grant no. 30870981).

\section{References}

1. de Martel C, Forman D and Plummer M: Gastric cancer: epidemiology and risk factors. Gastroenterol Clin North Am 42 : 219-240, 2013

2. Shridhar R, Almhanna K, Hoffe SE, et al: Increased survival associated with surgery and radiation therapy in metastatic gastric cancer: a Surveillance, Epidemiology, and End Results database analysis. Cancer 119: 1636-1642, 2013.

3. Guggenheim DE and Shah MA: Gastric cancer epidemiology and risk factors. J Surg Oncol 107: 230-236, 2013.

4. Zilberstein B, Jacob CE and Cecconello I: Gastric cancer trends in epidemiology. Arq Gastroenterol 49: 177-178, 2012.

5. Takaishi S, Okumura T, Tu S, et al: Identification of gastric cancer stem cells using the cell surface marker CD44. Stem Cells 27: 1006-1020, 2009.

6. Han ME, Jeon TY, Hwang SH, et al: Cancer spheres from gastric cancer patients provide an ideal model system for cancer stem cell research. Cell Mol Life Sci 68: 3589-3605, 2011.

7. Song Z, Yue W, Wei B, et al: Sonic hedgehog pathway is essential for maintenance of cancer stem-like cells in human gastric cancer. PLoS One 6: e17687, 2011.

8. Jemal A, Bray F, Center MM, Ferlay J, Ward E and Forman D: Global cancer statistics. CA Cancer J Clin 61: 69-90, 2011.

9. D'Angelo A, Bluteau O, Garcia-Gonzalez MA, et al: Hepatocyte nuclear factor 1alpha and beta control terminal differentiation and cell fate commitment in the gut epithelium. Development 137 1573-1582, 2010

10. Yuasa Y: Control of gut differentiation and intestinal-type gastric carcinogenesis. Nat Rev Cancer 3: 592-600, 2003.
11. Wilkinson NW, Howe J, Gay G, Patel-Parekh L, Scott-Conner C and Donohue J: Differences in the pattern of presentation and treatment of proximal and distal gastric cancer: results of the 2001 gastric patient care evaluation. Ann Surg Oncol 15: 1644-1650, 2008.

12. Houghton J, Morozov A, Smirnova I and Wang TC: Stem cells and cancer. Semin Cancer Biol 17: 191-203, 2007.

13. Liu G, Neumeister M, Reichensperger J and Yang RD: Therapeutic potential of human adipose stem cells in a cancer stem cell-like gastric cancer cell model. Int J Oncol 43: 1301-1309, 2013.

14. Cao H, Xu W, Qian H, et al: Mesenchymal stem cell-like cells derived from human gastric cancer tissues. Cancer Lett 274: 61-71, 2009.

15. Zhang S, Balch C, Chan MW, et al: Identification and characterization of ovarian cancer-initiating cells from primary human tumors. Cancer Res 68: 4311-4320, 2008.

16. Rappa G, Mercapide J, Anzanello F, et al: Growth of cancer cell lines under stem cell-like conditions has the potential to unveil therapeutic targets. Exp Cell Res 314: 2110-2122, 2008.

17. Fujii H,Honoki K, Tsujiuchi T, Kido A, Yoshitani K and Takakura Y: Sphere-forming stem-like cell populations with drug resistance in human sarcoma cell lines. Int J Oncol 34: 1381-1386, 2009.

18. Liu J, Ma L, Xu J, et al: Spheroid body-forming cells in the human gastric cancer cell line MKN-45 possess cancer stem cell properties. Int J Oncol 42: 453-459, 2013.

19. Zheng Q, Gong F, Xu Y, Zheng T and Ying M: Floating cells with stem cell properties in gastric cell line SGC-7901. Tumori 97: 393-399, 2011.

20. Wakamatsu Y, Sakamoto N, Oo HZ, et al: Expression of cancer stem cell markers ALDH1, CD44 and CD133 in primary tumor and lymph node metastasis of gastric cancer. Pathol Int 62: 112-119, 2012.

21. Chen T, Yang K, Yu J, et al: Identification and expansion of cancer stem cells in tumor tissues and peripheral blood derived from gastric adenocarcinoma patients. Cell Res 22: 248-258, 2012.

22. Duckworth CA, Clyde D and Pritchard DM: CD24 is expressed in gastric parietal cells and regulates apoptosis and the response to Helicobacter felis infection in the murine stomach. Am J Physiol Gastrointest Liver Physiol 303: G915-G926, 2012.

23. Zhang C, Li C, He F, Cai Y and Yang H: Identification of CD $44^{+} \mathrm{CD} 24^{+}$gastric cancer stem cells. J Cancer Res Clin Oncol 137: 1679-1686, 2011.

24. Wang M, Xiao J, Shen M, et al: Isolation and characterization of tumorigenic extrahepatic cholangiocarcinoma cells with stem cell-like properties. Int J Cancer 128: 72-81, 2011.

25. Chen W, Zhang X, Chu C, et al: Identification of CD44+ cancer stem cells in human gastric cancer. Hepatogastroenterology 60: 949-954, 2013.

26. Xue Z, Yan H, Li J, et al: Identification of cancer stem cells in vincristine preconditioned SGC7901 gastric cancer cell line. J Cell Biochem 113: 302-312, 2012.

27. Li Y, Zhao Y, Cheng Z, et al: Mesenchymal stem cell-like cells from children foreskin inhibit the growth of SGC-7901 gastric cancer cells. Exp Mol Pathol 94: 430-437, 2013.

28. Katsuno Y, Ehata S, Yashiro M, Yanagihara K, Hirakawa K and Miyazono K: Coordinated expression of REG4 and aldehyde dehydrogenase 1 regulating tumourigenic capacity of diffuse-type gastric carcinoma-initiating cells is inhibited by TGF- $\beta$. J Pathol 228: 391-404, 2012

29. Wu C, Xie Y, Gao F, et al: Lgr5 expression as stem cell marker in human gastric gland and its relatedness with other putative cancer stem cell markers. Gene 525: 18-25, 2013. 